\title{
Investigation of micro-structural phenomena at aggregate level in concretes using DEM
}

\author{
Michat Nitka ${ }^{1}$, and Jacek Tejchman ${ }^{1, *}$ \\ ${ }^{1}$ Faculty of Civil and Environmental Engineering, Gdańsk University of Technology, Gdansk, Poland
}

\begin{abstract}
This paper presents numerical analyses of concrete beams under three-point bending. The discrete element methods (DEM) was used to calculate fracture at the aggregate level. Concrete was described as a four-phase material, which was composed of aggregate, cement matrix, interfacial transitional zones (ITZs) and macro-voids. The beam micro-structure was directly taken from our experiments using x-ray micro-tomography. 3D simulations were carried out with real aggregate modelled as sphere clusters. Numerical results were compared with laboratory outcomes. The special attention was laid on the fracture propagation and some micro-structural phenomena at the aggregate level.
\end{abstract}

\section{Introduction}

The fracture process is a fundamental phenomenon in quasi- brittle materials like concrete. It is very complex since it consists of main cracks with various branches, secondary cracks and micro-cracks. During fracture, micro-cracks first arise in a hardening region on the stress-strain curve which change gradually during material softening into dominant distinct macroscopic cracks up to damage. The fracture process strongly depends upon a heterogeneous structure of materials over many different length scales, changing e.g. in concrete from the few nanometres (hydrated cement) to the millimetres (aggregate particles). In order to properly describe fracture, material micro-structure has to be taken into account since its effect on the global results is pronounced. At the meso-scale, concrete may be considered as a composite material wherein four important phases may be separated: cement matrix, aggregate, interfacial transition zones ITZs between the aggregate and cement matrix and macro-voids.

The main objective of this study is to investigate a complex fracture process in concrete beams under bending at the aggregate level under $3 \mathrm{D}$ conditions using the discrete element method (DEM) with angularlyshaped aggregate particles. Discrete models (if they are enough consistent) might progressively replace experimental tests to study the influence of concrete meso-structure (aggregates size, aggregate shape, aggregate roughness, aggregate/mortar volume, macro porosity, etc.) on the concrete behaviour. The disadvantages of DEM are: enormous computational cost and a difficult calibration procedure with respect to geometric and mechanical properties of ITZs. In the calculations, the concrete micro-structure was assumed based on 3D images by means of x-ray microtomography using the micro-tomograph Skyscan 117, which represents a new generation in high-resolution desktop x-ray micro-tomography systems [1]. The paper is a continuation of our research outcomes presented in [1], [2] which concerned the crack propagation in concrete beams under 2D conditions only.

\section{Experimental results}

Laboratory tests were carried out with notched concrete beams (height $80 \mathrm{~mm}$, depth $40 \mathrm{~mm}$ and length $320 \mathrm{~mm}$ with the notch $8 \mathrm{~mm}$ high and $3 \mathrm{~mm}$ wide) [1], [2]. Concrete was prepared as a mix of the cement matrix and aggregate (river sand and gravel). The mean aggregate diameter was $2 \mathrm{~mm}$ and maximum aggregate diameter was equal to $16 \mathrm{~mm}$. The aggregate volume was $75 \%$. Quasi-static three-point bending tests were performed with the controlled notch opening displacement rate (CMOD). The CMOD gauge with the length of $5 \mathrm{~mm}$ was located in the notch at the beam bottom. The maximum vertical force was equal to 2.18 $\mathrm{kN}$ for CMOD equal to $0.017 \mathrm{~mm}$.

Figure 1 shows the 3D $\mu \mathrm{CT}$ image of the cracked cuboidal specimen $\left(80 \times 50 \times 40 \mathrm{~mm}^{3}\right)$, cut out from the beam mid-part after one test [1]. The main crack was strongly curved mainly due to presence of aggregate particles. Its shape changed along the specimen depth in spite of the fact that 2D boundary value problem (plane stress) was considered. The discrete macro-crack mainly propagated through ITZs (which were the weakest phase in concrete) and sometimes through macro-voids. It might very rarely propagate through a single weak aggregate particle. The effect of macro-voids on the crack shape was small. The discrete crack was created by 
bridging the interfacial micro-cracks. It possessed many small branches. ITZs around aggregate particles were characterized by a very non-uniform porous structure and presence of separated small sand particles. They appeared mainly aggregate particles but sometimes they were also visible around larger cement matrix particles. The width of ITZs changed between 30 and $50 \mathrm{~lm}$. The width was independent of the aggregate diameter.

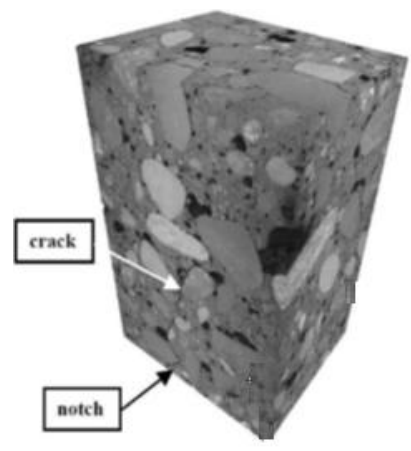

a)

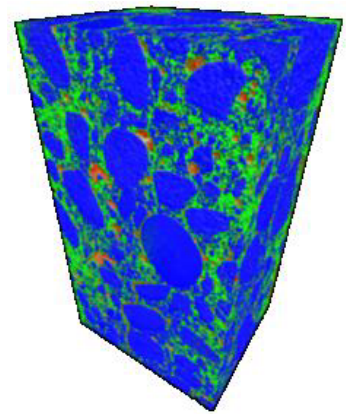

b)
Fig. 1. Images of cracked cuboidal specimens cut out from beam obtained by means of 3D $\mu \mathrm{CT}$ : a) general view and b) image with separated phase (blue colour - aggregate, green colour - cement matrix, red colour - crack and air void) [1].

\section{Numerical Results}

\subsection{Discrete element method}

DEM simulations shown in the paper were carried out using the open-source code YADE [3]. A linear normal contact model under compression was used. The interaction force vector representing the action between two elements in contact was decomposed into normal and tangential vectors. The normal and tangential forces were linked to the displacement through the normal $K_{n}$ and tangential $K_{\mathrm{s}}$ stiffness, respectively:

$$
\mathbf{F}_{\mathbf{n}}=K_{n} U \mathbf{N}
$$

and

$$
\mathbf{F}_{\mathbf{s}}=\mathbf{F}_{\mathrm{s}, \text { prev }}+K_{s} \Delta \mathbf{X}_{\mathbf{s}},
$$

where $U$ denote the overlap between elements, $\mathbf{N}$ denotes the normal vector at the contact point, $\Delta \mathbf{X}_{\mathbf{s}}$ is the increment of the relative tangential displacement and $\mathbf{F}_{\text {s,prev }}$ is the tangential force from the previous iteration. The contact forces satisfied the cohesive frictional MohrCoulomb equation:

$$
\left\|\mathbf{F}_{\mathbf{s}}\right\|-F_{s, \max }-\| \mathbf{F}_{\mathbf{n}}|| \tan \mu \leq 0 \text { (before contact breakage), }
$$

and

$$
\left\|\mathbf{F}_{\mathbf{s}}\right\|-\| \mathbf{F}_{\mathbf{n}}|| \tan \mu \leq 0 \text { (after contact breakage), }
$$

where $\mu$ is the inter-particle friction angle and $F_{s, \max }$ denotes the cohesive force between elements. The normal force might be negative down to the minimum tensile value of $F_{n, \min }$. The cohesive force and tensile force were assumed as a function of the cohesive stress $C$, tensile normal stress $T$ and element radius $R$ :

$$
F_{s, \max }=C \times R^{2} \quad \text { and } \quad F_{n, \min }=T \times R^{2} .
$$

Concrete was described as four-phase material which consisted of aggregate, cement matrix, interfacial transitional zones (ITZs) and macro-voids [1]. All aggregate particles (from $2 \mathrm{~mm}$ to $16 \mathrm{~mm}$ ) were modelled as grain clusters composed of pure spheres connected to each other as rigid bodies. All aggregate particles (larger than $2 \mathrm{~mm}$ ) included ITZs. ITZs were solely modelled as the contacts between aggregate and cement matrix (thus they had no a physical width). Cement matrix was simulated with spheres of the diameter 0.5-2 $\mathrm{mm}$ (without ITZ). Macro-voids were assumed as empty spaces. The remaining beam region (outside the meso-part close to the notch) was modelled with spheres with the diameter of $2-8 \mathrm{~mm}$ in order to reduce calculation time (Fig.2).

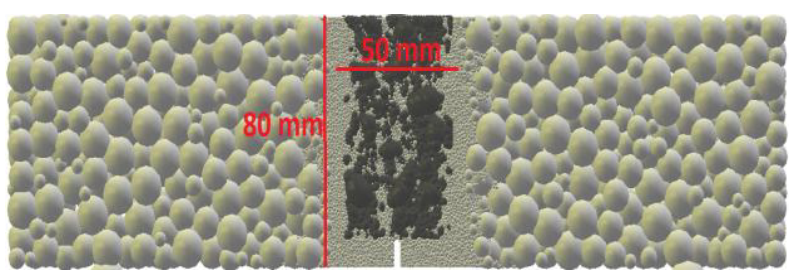

Fig. 2. Front face of concrete beam with meso-region of $50 \times 80 \times 40 \mathrm{~mm}^{2}$ close to notch (dark grey corresponds to aggregate and light grey is related to cement matrix).

The following parameters of the elastic modulus of grain contact $E$, cohesion $C$ and tensile strength $T$ were assumed in DEM calculations: $E_{c m}=29.2 \mathrm{GPa}$, $C_{c m}=140 \mathrm{MPa}$ and $T_{c m}=25 \mathrm{MPa}$ for the cement matrix, $E_{i t z}=20.4 \mathrm{GPa}, C_{i t z}=100 \mathrm{MPa}$ and $T_{i t z}=17.5 \mathrm{MPa}$ for ITZs and $E_{\text {macro }}=36.1 \mathrm{GPa}, C_{\text {macro }}=140 \mathrm{MPa}$, and $T_{\text {macro }}=25 \mathrm{MPa}$ for the region outside the meso-region. Thus, ITZs were the weakest phase. The calibration procedure was based on uniaxial compressive and tensile tests [4]. The remaining DEM parameters were constant for all phases and regions: Poisson's ratio of grain contact $\nu=0.2$, inter-particle friction angle $\mu=18^{\circ}$, damping parameter $\alpha=0.08$ and mass density $\rho=2600 \mathrm{~kg} / \mathrm{m}^{3}$. The calculations were performed under quasi-static conditions (inertial number was $I<10^{-3}$ ). The entire beam included about $155^{\prime} 000$ spherical elements with $150^{\prime} 000$ in the meso-region (20`000 for aggregate and 130 '000 for cement matrix).

\subsection{Numerical 3D results}

The evolution of the resultant vertical force $F$ against CMOD from DEM is shown in Fig.2 as compared to the experimental curve. The computed maximum vertical force was $F_{\text {max }}=2.19 \mathrm{kN}$ for $\mathrm{CMOD}=0.017 \mathrm{~mm}$ and was in agreement with the experimental value. The calculated residual force was however too high. 


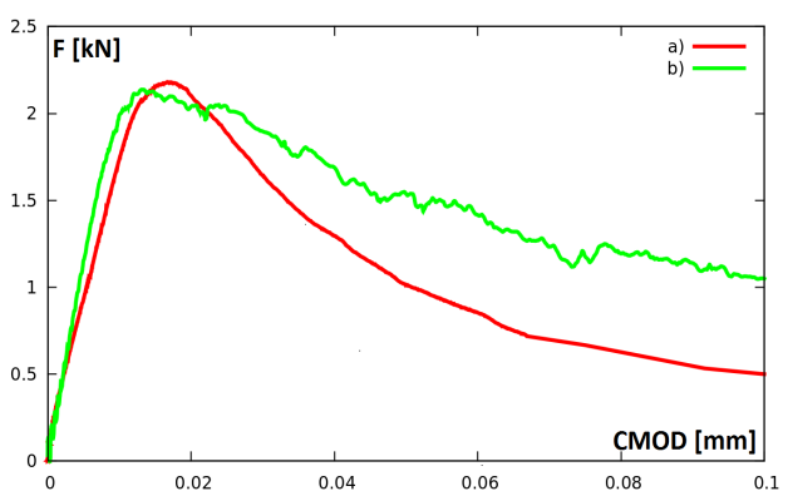

Fig. 3. Evolution of vertical force $F$ against displacement CMOD in beam under 3-point bending: a) experimental curve and b) 3D DEM.

In Fig. 4, the numerical final crack path was shown for $\mathrm{CMOD}=0.1 \mathrm{~mm}$ in 2 different vertical cross-sections.
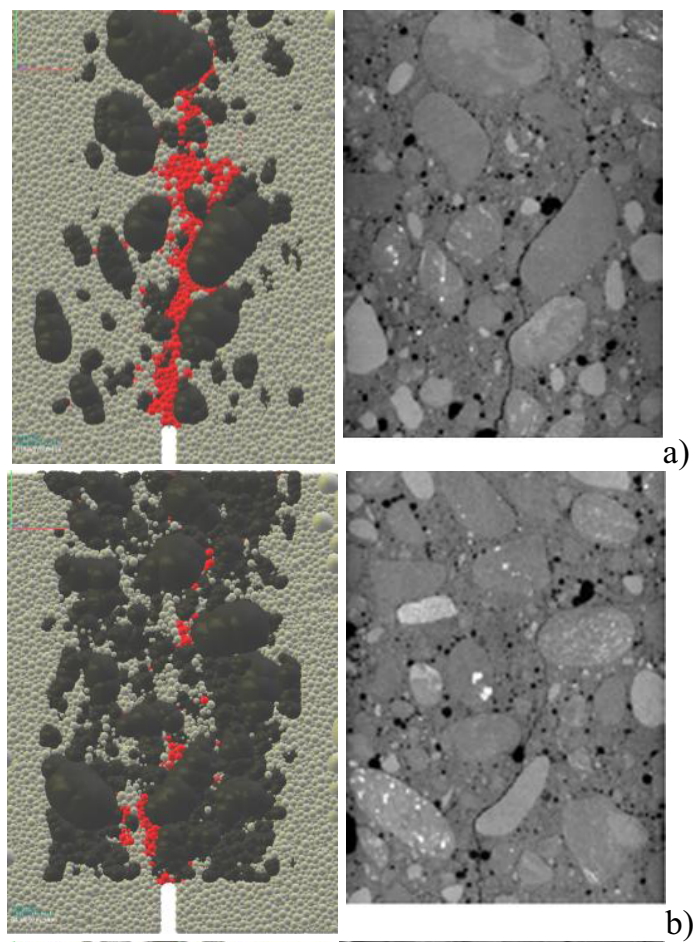

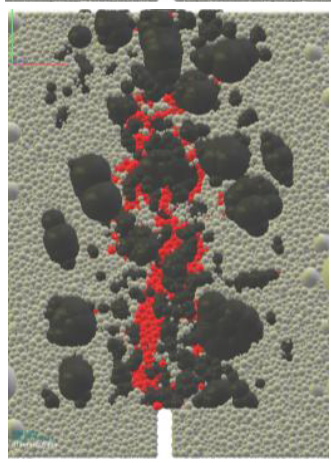

A)

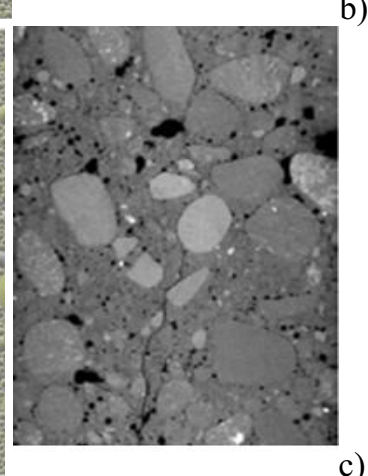

B)
Fig. 4. Final crack path in concrete beam above notch in 2 different cross-sections from: A) 3D DEM and B) experiment (10 $\mathrm{mm}$ from beam front side (a), beam mid-depth (b) an $10 \mathrm{~mm}$ from beam rear side (c), red colour denotes cement spheres with broken contacts, dark grey denotes aggregate and light grey denotes cement matrix).
The crack propagation was similar as in experiments. The crack was strongly curved along height and depth due to a stochastic distribution of aggregate (Fig. 5). The crack high varied along the beam depth from $52 \mathrm{~mm}$ up to $58 \mathrm{~mm}(50-55 \mathrm{~mm}$ in the experiment). The width of the crack varied between $0.05-0.1 \mathrm{~mm}$ above the notch up to $0.01 \mathrm{~mm}$ at the crack tip.

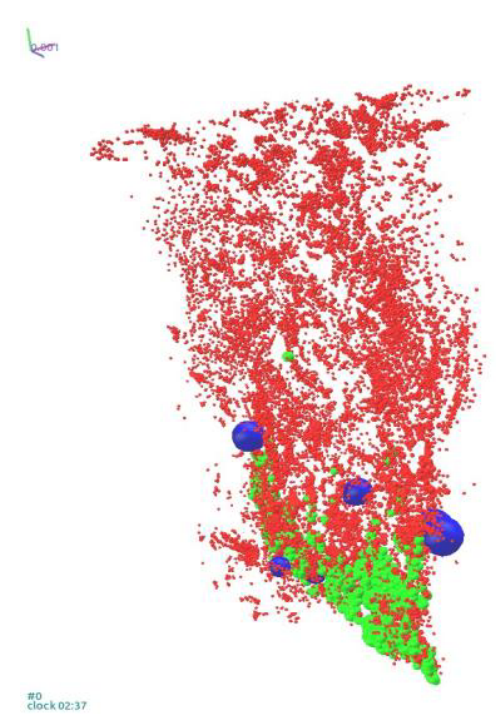

Fig. 5. Calculated cracked region (empty space) above notch from 3D DEM for $\mathrm{CMOD}=0.1 \mathrm{~mm}$ (blue colour corresponds to crack width larger than $0.2 \mathrm{~mm}$, green colour to crack width $0.05-0.2 \mathrm{~mm}$ and red colour to crack width smaller than $0.05 \mathrm{~mm})$

The evolution of the broken contact number is shown in Fig. 6a. The initial contact number in the beam microregion was about 640 '000 with the coordination number of about 10. The breakage started early for about $\mathrm{CMOD}=0.007 \mathrm{~mm}$ (the peak force was for $\mathrm{CMOD}=0.017 \mathrm{~mm})$. Finally, more than 7'000 contacts were broken that corresponded to $1 \%$ of all existing contacts. The global void ratio of the meso-region initially showed contractancy and later dilatancy for CMOD $>0.01 \mathrm{~mm}$ (Fig. 6b).

The evolution of the normal contact force network between spheres is demonstrated in Fig. 7 (the red colour corresponds to the high compressive normal contact forces (higher than the average ones) and blue colour to the high tensile normal contact forces (lower than the average ones). The remaining contact forces were marked in green. The line thickness denotes the magnitude of the normal contact force. The external vertical force $F$ was transmitted via a network of normal contact forces which formed pronounced force chains. Their distribution was strongly non-uniform due to the grain rearrangement after the crack development. The maximum compressive contact force was about $30 \mathrm{~N}$ and the maximum tensile contact forces was about $-20 \mathrm{~N}$. Some pronounced compressive forces also existed in the damage zone parallel to the crack due to shear along a rough crack surface (aggregate interlocking). 


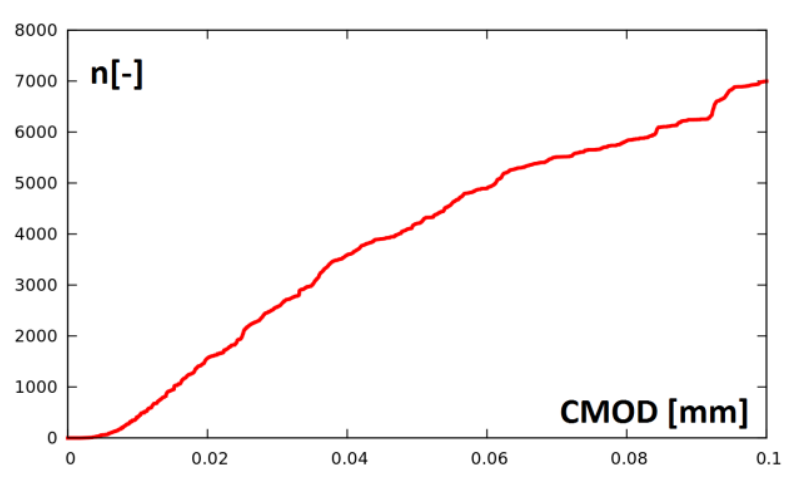

a)

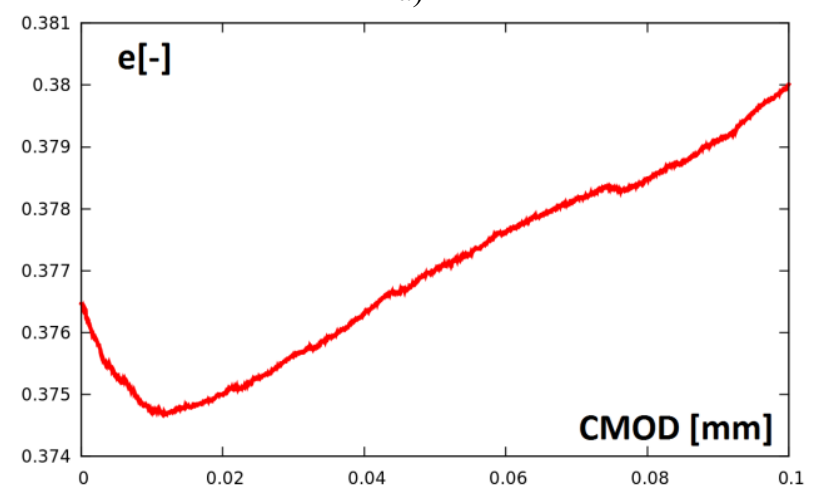

b)

Fig. 6. DEM analyses: a) evolution of broken contacts and b) evolution of global void ratio in 3D meso-region.

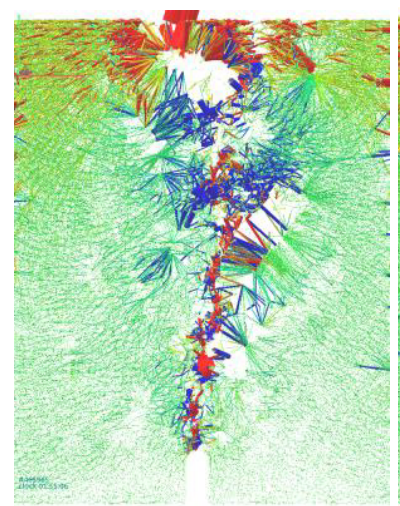

a)

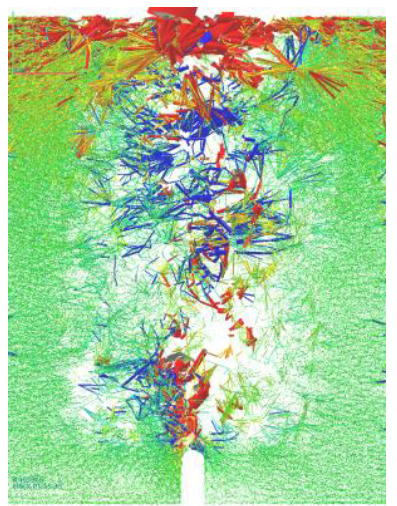

b)

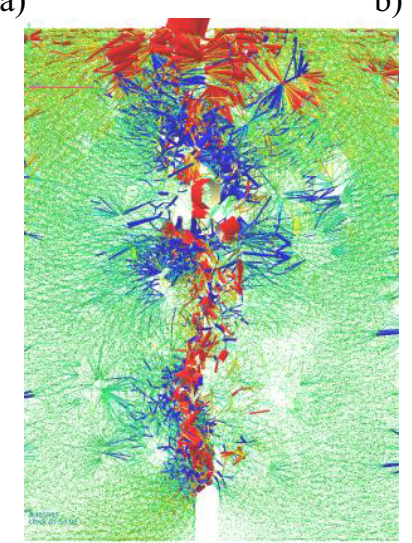

c)

Fig. 7. Distribution of normal contact forces between elements in 3 different cross-sections: a) $10 \mathrm{~mm}$ from beam front side, b) beam mid-depth and c) $10 \mathrm{~mm}$ from beam rear side (red colour denotes high compressive forces, blue colour - high tensile forces and green colour denotes remaining forces).

\section{Conclusions}

The 3D calculation results for four-phase concrete at the aggregate level using the discrete element model showed satisfactory agreement with experimental observations. They evidently showed that DEM was able to realistically capture the beam strength and fracture in concrete beams at the macro-level under condition that the real aggregate shape and location were mapped. DEM realistically followed a fracture process including the occurrence of micro- and macro-cracks during their onset, formation and propagation (including phenomena of crack bridging, crossing, branching and closing). In addition, it allowed for investigating different interesting micro-structural events occurring at the aggregate level (e.g. force chains, contact breakage, particles rotations).

The experimental discrete macro-crack above the notch was strongly curved and depended on the concrete micro-structure. It was created by bridging the interfacial micro-cracks. It mainly propagated through the weakest contact zones (ITZs) between the cement matrix and aggregate. The width of ITZs varied between 30 and $50 \mu \mathrm{m}$. Their porosity was strongly uniform. The crack rarely propagated through weak aggregate particles. Some small crack branches were also visible. The crack shape was different with the beam depth. Single microcracks also occurred far beyond the macro-crack.

It was found that the mechanical properties of ITZs which were cracks' attractors had a pronounced influence on the crack strength and macro-cracking. The weaker strength of ITZs and their higher number increased the beam ductility (due to a longer crack propagation way).

The external load was transmitted via a network of normal contact forces which formed force chains of a different intensity. The compressive normal contact forces (connected to the tangential contact forces) also developed along the macro-crack due to aggregate interlocking.

The research work has been carried out within the project: "Innovative ways and effective methods of safety improvement and durability of buildings and transport infrastructure in the sustainable development" financed by the European Union (POIG.01.01.02-10-106/09-01) and the project "Experimental and numerical analysis of coupled deterministic-statistical size effect in brittle materials" financed by the National Research Centre NCN (UMO-2013/09/B/ST8/03598).

\section{References}

1. Ł. Skarżyński, J. Tejchman, Strain 52, 26-45 (2015)

2. Ł. Skarzyński, M. Nitka, J. Tejchman, Engng. Fract. Mech. 147, 13-35 (2015)

3. J. Kozicki, F. Donze, Computer Methods in Applied Mechanics and Engineering 197, 4429-4443 (2008)

4. M. Nitka, J. Tejchman, Granular Matter 17, 145-164 (2015) 